\title{
Diversity in conserved genes in tomato
}

\section{Allen Van Deynze*1, Kevin Stoffel ${ }^{1}$, C Robin Buell2,3, Alexander Kozik ${ }^{4}$, Jia Liu ${ }^{2}$, Esther van der Knaap ${ }^{5}$ and David Francis ${ }^{5}$}

\begin{abstract}
Address: ${ }^{1}$ Seed Biotechnology Center, University of California, 1 Shields Ave., Davis, CA, USA, 2The Institute for Genomic Research, 9712 Medical Center Dr, Rockville, MD, USA, ${ }^{3}$ Department of Plant Biology, Michigan State University, 166 Plant Biology, East Lansing, MI, USA, ${ }^{4}$ Genome and Biomedical Sciences Facility, University of California, 1 Shields Ave., Davis, CA, USA and ${ }^{5}$ Department of Horticulture and Crop Science, The Ohio State University/OARDC, 1680 Madison Ave, Wooster, OH, USA

Email: Allen Van Deynze* - avandeynze@ucdavis.edu; Kevin Stoffel - kmstoffel@ucdavis.edu; C Robin Buell - buell@msu.edu; Alexander Kozik - akozik@atgc.org; Jia Liu - jliu@jcvi.org; Esther van der Knaap - vanderknaap.1@osu.edu; David Francis - francis.77@osu.edu * Corresponding author
\end{abstract}

Published: 18 December 2007

BMC Genomics 2007, 8:465 doi:10.1/86/147|-2164-8-465
Received: 6 July 2007

Accepted: 18 December 2007

This article is available from: http://www.biomedcentral.com/I47I-2/64/8/465

(C) 2007 Van Deynze et al; licensee BioMed Central Ltd.

This is an Open Access article distributed under the terms of the Creative Commons Attribution License (http://creativecommons.org/licenses/by/2.0), which permits unrestricted use, distribution, and reproduction in any medium, provided the original work is properly cited.

\begin{abstract}
Background: Tomato has excellent genetic and genomic resources including a broad set of Expressed Sequence Tag (EST) data and high-density genetic maps. In addition, emerging physical maps and bacterial artificial clone sequence data serve as template to investigate genetic variation within the cultivated germplasm pool with the goal to manipulate agriculturally important traits. Unfortunately, the nearly exclusive focus of resource development on interspecific populations for genetic analyses and diversity studies has left a void in our understanding of genotypic variation within tomato breeding programs that focus on intra-specific populations. We describe the results of a study to identify nucleotide variation within tomato breeding germplasm and mapping parents for a set of conserved single-copy ESTs that are orthologous between tomato and Arabidopsis.
\end{abstract}

Results: Using a pooled sequencing strategy, 967 tomato transcripts were screened for polymorphism in 12 tomato lines. Although intron position was conserved, intron lengths were 2fold larger in tomato than in Arabidopsis. A total of I,487 single nucleotide polymorphisms and 282 insertion/deletions were identified, of which 579 and 206 were polymorphic in breeding germplasm, respectively. Fresh market and processing germplasm were clearly divergent, as were Solanum lycopersicum var. cerasiformae and Solanum pimpinellifolium, tomato's closest relatives. The polymorphisms identified serve as marker resources for tomato. The COS is also applicable to other Solanaceae crops.

Conclusions: The results from this research enabled significant progress towards bridging the gap between genetic and genomic resources developed for populations derived from wide crosses and those applicable to intra-specific crosses for breeding in tomato.

\section{Background}

Tomato (Solanum lycopersicum, $\mathrm{Sl}$ ) has rich genetic and genomic resources including comprehensive databases of Expressed Sequence Tags (ESTs), Bacterial Artificial Chro- mosome (BAC) libraries, and genetic and comparative maps which are in the process of being linked to a physical map and eventually the euchromatic genomic sequence. These resources serve as template to study 
genetic variation and to manipulate agricultural traits. Current genetic maps for tomato include 2,200 Restriction Fragment Length Polymorphisms (RFLPs), Cleaved Amplified Polymorphic Sequences (CAPs), and Simple Sequence Repeats (SSRs), as well as emerging genetic resources which include a comparative map with Arabidopsis of over 500 Conserved Orthologous Set (COS) markers $[1,2]$. These maps were derived from populations that were developed between wild relatives (various Solanum species) and cultivated varieties. This approach maximizes genetic variation and has led to the discovery and introgression of novel alleles for disease resistance [3]and fruit traits $[4,5]$ into cultivated germplasm. However, the nearly exclusive focus on wide crosses has left a void in our knowledge and ability to manipulate other traits of agricultural importance within cultivated tomato. There is a lack of molecular markers that detect nucleotide polymorphisms among elite breeding lines. With the exception of genes that were introgressed from wild species, the majority of the breeding efforts in tomato are derived from elite-by-elite intra-specific crosses, resulting in consistent improvement for yield and fruit quality [6]. Increasing molecular marker density to facilitate the evaluation and analysis of elite-by-elite breeding populations is highly desirable.

Given the discrepancy of resources available between inter-specific and intra-specific crosses, we sought to test the feasibility of a large-scale screen for identifying polymorphisms within cultivated tomato lines. Previous examinations of EST databases suggested that Single Nucleotide Polymorphisms (SNPs) could be detected within tomato EST databases $[7,8]$. Single nucleotide polymorphisms, are highly abundant in plant species. They can be associated with known genomic sequences and are bi-allelic [9]. Current technology allows SNPs to be assayed cost-effectively for applications in breeding programs and genetic studies. Analysis of tomato EST sequences resulted in only limited information on SNPs that occurred within cultivated tomato germplasm. In a previous study, the GenBank EST database was mined for SNPs which resulted in 101 putative SNPs, 83 of which were confirmed empirically. Of the 83 validated SNPs, $53 \%$ proved useful within cultivated germplasm [8]. A similar study using an algorithm that considered sequence context, but not sequence redundancy, identified 764 putative SNPs from a similar dataset from which only $27 \%(28 / 103)$ of the putative SNPs tested could be validated experimentally [7]. The paucity of SNPs within the ESTs can be attributed to low rate of polymorphism within gene coding regions of tomato and a limited sampling of genotypes in the databases, and shallow sampling within each of these genotypes. The current resources are thus insufficient for breeding studies.
Extrapolation of these experimentally defined SNP rates to the gene and genome level projects a rate of 1 SNP every 4000-8500 bp of coding sequence. Variation in genes has been shown to be highest in untranslated regions (UTRs) [9] and within introns in which nucleotide diversity is more tolerated compared to protein coding regions [10]. Often, intron position is conserved between species [11], therefore one can leverage the Arabidopsis genome sequence and orthologous EST sequences from different species to predict intron position. Thus, a strategy for SNP discovery is to utilize available EST sequences and design primers that flank introns or amplify the 3 ' or 5 ' UTR from genomic DNA.

Genomic resources of Arabidopsis can be used to identify single-copy genes in tomato which in turn can be used as template to develop DNA markers. A COS is defined as a set of genes that are conserved throughout plant evolution in both sequence and copy number $[12,13]$. Sequences from a single-copy COS of Arabidopsis can be used as a reference to bridge relationships across genes identified through ESTs in crops. A COS in tomato (SGN COS I) was identified by comparing the set of unique gene sequences in tomato ( $\mathrm{Sl}$ only) to all translated proteins in the fully sequenced genome of Arabidopsis [12,14]. Approximately $10 \%$ of the 27,000 tomato unigene sequences meet the defined COS criteria. The SGN COS I set was further refined to include pepper, potato, eggplant and coffee for a combined COS (SGN COS II) of 2,869 unigenes [14] across five species. Southern hybridization revealed that $85 \%$ of the COS tested are single-copy sequences in tomato and 13 other plant species from the Solanaceae, Asteraceae, Leguminosae, Cucurbitaceae, Rosaceae, Brassicaceae, Poaceae, and Malvaceae families [12]. Using different approaches, we identified 2,185 sequences as a COS between Arabidopsis and tomato, lettuce, sunflower, soybean and maize, of which 1,704 are represented in tomato. The parameters for developing the latter dataset are described at Compositae Genome Project Database [15] and summarized in Results and Discussion. A COS database provides a unique resource that can be applied to marker development for crop improvement and assist in comparative genomic analyses. In this study, we developed primers flanking predicted introns in the tomato COS and used these to amplify genomic DNA from several tomato genotypes. Amplified loci were pre-screened to identify sequence variation using pools of DNA from diverse genotypes. Loci identified as polymorphic were subsequently sequenced for 12 genotypes representing closely related wild relatives, processing tomato germplasm adapted to arid and humid growing conditions, fresh-market germplasm, and heirloom germplasm. The resulting sequence data, described in the current paper, is intended to greatly increase the availability of DNA-based markers for intra-specific tomato crosses and to character- 
ize and manipulate traits important for tomato breeding populations.

\section{Results and Discussion Conserved Orthologous Sets}

Our goal is to identify and characterize sequence variation within cultivated tomato in genes that can be used in genetic analysis, functional genomic studies, and breeding efforts. By focusing on a COS as the basis for marker discovery, we also intended to develop a sequence resource representing single-copy loci that can be assayed reproducibly for genetic analysis within $S l$ and in other related species. The challenge to identify a COS with incomplete EST datasets is to accurately predict singlecopy and orthologous genes that do not represent paralogs. EST databases are highly redundant and unigene sets of assembled ESTs tend to over-represent the complement of transcripts. For example, both the Arabidopsis and Rice EST databases (> 700,000 ESTs) represent about 150,000 unigenes compared to less than 30,000-45,000 predicted genes with whole genome sequence [16]. The difference in number is attributable to allelic variation, sequencing errors, alternative splice forms, and paralogs which can not be easily addressed in the assembly of transcripts in the absence of a complete genome and sequence of full length cDNAs.

Designing a COS involves decisions regarding stringency of match, order of comparisons, and definitions of orthologs $[14,17]$. One approach taken to identify a COS is to compare unigene sets anchored to single-copy loci in Arabidopsis and generate reciprocal best matches for pairwise comparisons among several species and Arabidopsis [14]. With this strategy, only unigenes with a single best match to Arabidopsis are considered. In tomato, this approach resulted in a COS of 2,587 sequences (SGN COS II) which was further used to design primers with homology across species to amplify loci in tomato, pepper, potato and coffee. Fifteen loci were sequenced across these species for phylogenetic analysis. The strategy resulted in a COS that over-represented genes involved in organelle and chloroplast-associated proteins and DNA/ RNA metabolism, and under-represented cell wall, transcription factors, protein kinases and signal transduction peptides [14]. We used a more conservative approach to identify a COS by first identifying single-copy proteins in Arabidopsis using a "BLASTP all-vs-all" search and then comparing single-copy Arabidopsis genes to all tomato ESTs (all S. lycopersicum, Solanum habrochaites and Solanum pennellii ESTs in GenBank in 2003) using TBLASTX. From these results, we identified the single best tomato EST match (usually the longest) to an Arabidopsis sequence and performed a BLASTN search against the Arabidopsis genome to verify the hit. The 1,704 tomato ESTs that had a single hit in Arabidopsis were included in a COS which we designate UCD COS to distinguish it from the SGN COS I and SGN COS II. As the number of ESTs used to create the UCD COS was less than that used in the SGN COS II created later we expect the UCD COS to have less sequences than SGN COSII. By selecting a single EST for each contig, incorrect assemblies of paralogs and errors due to alternative splicing are avoided [15].

For transparency, we cross-referenced all UCD Tomato COS to the SGN COS II integrated dataset for Solanum species (Table 1, See Additional file 1,[14]). The 1,704 UCD COS have single hits with a BLAST expect value cutoff of e-20 over $80 \%$ of the UCD contig length to 1,611 SGN Unigenes (SGN build Tomato200607,[1]), 857 tomato, 729 potato, 418 pepper and 442 coffee COS II markers. The UCD COS represents 847 unigenes that are not in SGN COS II which expands the total COS by 33\% from 2,587 (SGN COS II) to 3,434 unigenes (See Additional file 1). To further investigate the application of the UCD COS, we evaluated the ability of 96 primers designed solely for tomato to amplify single-copy sequences in pepper and eggplant. Primers designed from loci with COS represented in at least two species and Arabidopsis were more likely to amplify single-copy loci in eggplant (58 vs. $38 \%$ ) and pepper ( 44 vs. $25 \%$ ) than those found only in the tomato COS. Only two primers in eggplant and four primers in pepper amplified multiple fragments (data not shown). This verifies the utility of conserved sequence in species with less genomic resources. Although the independent approaches to COS development are similar, we have shown that they verify each other, yet can be complementary. Nevertheless, both COS sets empirically yielded genomic resources to characterize putative single-copy conserved genes.

\section{The Size of Introns in Tomato}

We designed primers spanning putative introns from 1,112 ESTs in the UCD COS. Greater than one primer set was designed for some contigs. Intron positions were predicted by comparing tomato COS ESTs to Arabidopsis protein sequence (See Materials and Methods) [18]. With

Table I: Number of UCD COS in common with SGN COSII.

\begin{tabular}{lr}
\hline Species & Total' $^{\prime}$ \\
\hline Tomato & 857 \\
Tomato map & 122 \\
Mapped SNPs & 29 \\
Mapped indels & 22 \\
Mapped SSRs & 6 \\
Potato & 729 \\
Pepper & 418 \\
Coffee & 442 \\
\hline
\end{tabular}

'Homology based on single hits of UCD COS at e-20 with matching over $80 \%$ of the length of the SGN COS II 
these primers, 967 loci were amplified in 12 tomato lines (Table 2). Consequently, forward and reverse sequence across the resulting amplicons generated 1,241 predicted introns from 1102 unique contigs (1.33 contigs/locus) derived from 825 primer sets (Fig. 1). Greater than one contig/locus resulted when forward and reverse sequences did not overlap when primers spanned large introns. A total of 825 of the 967 loci yielded BLAST hits to the original COS EST database. The loci with poor BLAST results were due to the fact that the primers were located close to the intron ( $>100 \mathrm{bp}$ ) resulting in the near lack of sequence to COS EST coding sequence. BLAST of genomic sequence data generated from PCR amplicons back to the corresponding EST allowed intron size to be estimated for each contig by subtracting the last position (3') of a query from the corresponding first position $\left(5^{\prime}\right)$ of the next match. Intron positions were accurately predicted greater than $98 \%$ of the time based on differences between expected and predicted fragment sizes on agarose gels and sequence analysis. The experimentally estimated intron size ranged from 63 to 1469 bp. For 310 loci, the intervening region was either too large to form a contig from forward and reverse sequence pairs, or had no hits to ESTs. By assuming that the 310 pairs that did not assemble into a contig were comprised of introns that were at least 1,250 bp in length, the predicted average intron length in tomato would be $543 \mathrm{bp}$. These estimates are consistent with the limited information in tomato and suggest a twofold intron size increase in tomato relative to Arabidopsis $[14,19,20]$. Furthermore, 263 EST sequences $(23.8 \%)$ have more than one intron. A comparison of our intron sequences to the tomato repeat database (unirepeats.30.20060602,[21]) using BLAST indicated that there are few defined repeats in these tomato introns. Only 26 introns had homology to repeats in the database; one defined as a Long Terminal Repeat, another as DNA/ Mutator and the rest are annotated as unknown repeats.

\section{Diversity of Tomato Introns}

Pooling of samples has been used to efficiently screen BAC libraries for presence of sequences, identify novel mutations through TILLING, estimate gene frequencies in populations, and identify variants for specific traits through bulk-segregant analysis. We extended this idea to pre-screen DNA sequences for polymorphism in germplasm. This approach is particularly efficient when screening narrow germplasm pools such as those found in the majority of agricultural crops, especially self-pollinated species such as tomato, which have gone through genetic bottlenecks during domestication and selection. Singlestranded conformation polymorphism was suggested as a tool for screening loci for polymorphism [22]. In our experience, this approach was only effective for amplicons smaller than $250 \mathrm{bp}$ (data not shown), which is too small for efficient marker discovery in species with limited diversity and SNP density.

To develop an efficient strategy for SNP discovery in crops with narrow germplasm, we empirically determined that sequencing pools of four lines can effectively be employed to screen for polymorphisms (see Material and Methods). At first, we evaluated the variation among breeding lines and between species by sequencing across introns of 30 COS loci in 12 select tomato lines (See Table 2). This approach allowed us to refine the pooling strategy and establish a base line for polymorphism rate. This initial sequencing effort yielded $12(40 \%)$ polymorphic loci. Amplicons for the remaining 937 loci were then sequenced in pools (See Materials and Methods) for polymorphism discovery. Of these loci, 309 (33\%), showed a potential polymorphism within or between pools (Table 3). As the goal was to select for loci that were polymorphic in breeding germplasm, only $\mathrm{Sl}$ lines were included in the pools for screening. The pooled strategy had an accuracy rate of $98 \%$, with 302 loci showing polymorphisms in at least one line when sequenced individually (Table 3, See Additional file 1). Therefore, we reduced

Table 2: Summary of lines used for sequencing.

\begin{tabular}{|c|c|c|c|c|c|}
\hline Line & Market Class & Description & Use & Included in pool & Reference \\
\hline M82 & Processing & Inbred & Introgression line mapping & Pool I & [3I] \\
\hline O 8245 & Processing & Inbred, FI parent & Color and lycopene QTL & Pool I & {$[3,32]$} \\
\hline 088119 & Processing & Inbred, FI parent & Disease resistance, IBC pop & Pool I & [33] \\
\hline Sun 1642 & Processing & Inbred & Fruit shape QTL mapping & Pool I & [34] \\
\hline Heinz 1706 & Processing & Inbred & Donor for whole genome sequencing & Pool 2 & {$[35,36]$} \\
\hline O 9242 & Processing & Inbred, FI parent & Color and lycopene QTL & Pool 2 & {$[3,8]$} \\
\hline NC84I73 & Fresh Market & Inbred, FI parent & Disease resistance QTL & Pool $2 \& 3$ & [37] \\
\hline Fla7600 & Fresh Market & Inbred & Disease resistance, IBC pop & Pool $2 \& 3$ & [33] \\
\hline Ha7998 & Breeding line & Inbred & Disease resistance, IBC pop & Pool 3 & [33] \\
\hline San Marzano & Heirloom & & & Pool 3 & \\
\hline PIII4490 & NA & S. lycopersicum var. cerasiformae & Disease resistance, IBC pop & & [33] \\
\hline LAI589 & NA & S. pimpinellifolium & Mapping reference & & [34] \\
\hline
\end{tabular}




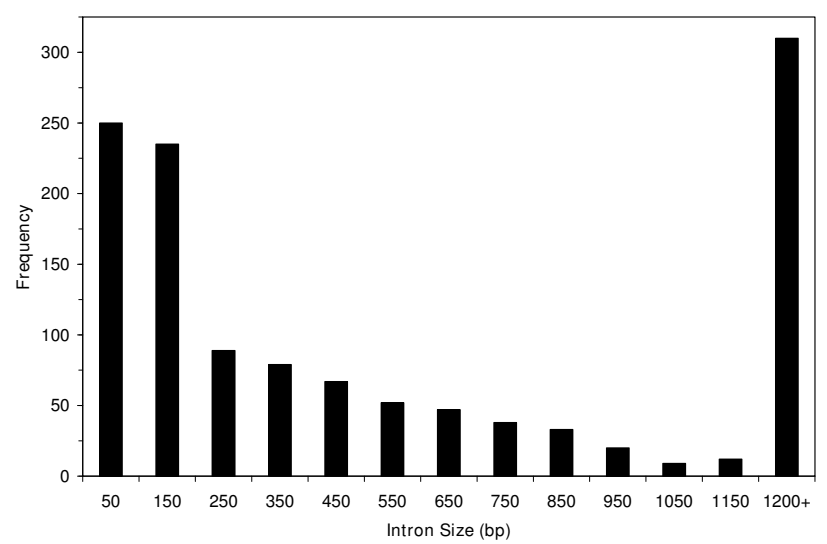

Figure I

Distribution of intron sizes of 825 COS ESTs in tomato.

the sequencing efforts three-fold by using the pooled genotypes strategy.

By using a pooling and re-sequencing strategy, we uncovered variation in ten domesticated breeding lines of $S l$, one wild cherry tomato (S. lycopersicum var. cerasiformae, Slc) and the progenitor species, S. pimpinellifolium (Sp) across predicted introns in 302 of 967 ESTs (Tables 3 and $4)$. On average, we assayed a minimum of 985 high quality base pairs per locus (range 62-2262 bp) with an average sequence read length of $619 \mathrm{bp}$ (see Additional file 1 ). Overall, 1,487 SNPs were identified, of which 579 from 162 loci were polymorphic in Sl breeding germplasm; 586 between Slc and other lines; and 1,121 between Sp and other lines (See Additional file 1). Interestingly, 402 SNPs were present within processing tomato lines and 168 within fresh market lines, but 400 differentiated the processing from fresh market classes indicating diversity among gene pools (Table 4, See Additional file 1). In the same samples, 282 indels were also detected in 153 contigs with 206 indels from 122 loci in breeding germplasm; 154 between Slc and other lines; and 152 between Sp and
Table 3: Summary statistics for sequence database.

\begin{tabular}{lcc}
\hline & Number & Percentage \\
\hline Primers Tested & 1268 & \\
Amplified single copy & 967 & 76 \\
Tested in pooled sequencing & 937 & \\
Polymorphic in pools & 309 & 33 \\
Sequenced in lines & 308 & \\
Polymorphic & 302 & 98 \\
\hline
\end{tabular}

other lines. Fresh market and processing tomatoes differed by 45 indels (Table 2, See Additional file 1). Only 13 loci had indels and no SNPs. Polymorphisms due to SNPs or indels range from 8 to $24 \%$ between any two breeding lines (See Additional file 1). SSR signatures ( 2 to 4 nucleotide repeats) were identified in 44 loci of which 12 were polymorphic and 21 unique to the indel and SNP databases (See Additional file 1). Ten SSRs were scored as indels as well. Overall, 967 EST loci have sequence information within introns, of which 302 are polymorphic. All sequences are available at GenBank (ET165605 to ET166001)[23].

Initial work [8] showed that SNP frequency is approximately $1 / 8500 \mathrm{bp}$ when surveying coding sequence (ESTs) between TA496 and Rio Grande. The work was based on identifying SNPs between only two sequenced genotypes, and therefore is an underestimate of the variation across a larger set of lines. Within the $10 \mathrm{Sl}$ breeding lines in the current project, $16.8 \%$ (162 of the 967 loci tested, See Additional file 1) of the loci were polymorphic with a SNP frequency of 1/1647 bp, a 5.2 fold increase over coding sequence reported [8]. Although, these datasets are not directly comparable due to both the number and actual lines and loci being compared, they are in line with those reported in the literature. Previous studies on a limited number of loci have reported a 2.7 to 5.3 fold $[8,14,24]$ increase in SNP frequency in tomato introns vs. exons.

Table 4: Summary statistics for SNPs and indels in introns of 12 lines of tomato.

\begin{tabular}{|c|c|c|c|c|c|c|c|c|c|c|}
\hline & $\begin{array}{l}\text { Loci with } \\
\text { SNPs }\end{array}$ & $\begin{array}{l}\text { Number } \\
\text { of SNPs }\end{array}$ & $\begin{array}{c}\% \text { Loci } \\
\text { with SNPs }\end{array}$ & SNPs/locus & Bases/SNPI & $\begin{array}{l}\text { Loci with } \\
\text { indels }^{2}\end{array}$ & $\begin{array}{l}\text { Number } \\
\text { of indels }\end{array}$ & $\begin{array}{c}\% \text { Loci } \\
\text { with indels }\end{array}$ & $\begin{array}{l}\text { Indels/ } \\
\text { locus }\end{array}$ & $\begin{array}{l}\text { Bases/ } \\
\text { indel' }^{\prime}\end{array}$ \\
\hline Total & 302 & 1487 & 31.2 & 4.9 & 641 & 153 & 282 & 16 & 1.8 & 3378 \\
\hline S. lycopersicum & 243 & 980 & 25.1 & 4.0 & 973 & 139 & 248 & 14 & 1.8 & $384 I$ \\
\hline Breeding lines $^{3}$ & 162 & 579 & 16.7 & 3.6 & 1647 & 122 & 206 & 13 & 1.7 & 4624 \\
\hline $\begin{array}{l}\text { US Fresh } \\
\text { Market }\end{array}$ & 53 & 168 & 5.5 & 3.2 & 5675 & 106 & 158 & II & 1.5 & 6028 \\
\hline Processing & 121 & 402 & 12.5 & 3.3 & 2372 & 118 & 193 & 12 & 1.6 & 4935 \\
\hline var cerasiformae & 161 & 586 & 16.6 & 3.6 & 1627 & 98 & 154 & 10 & 1.6 & 6185 \\
\hline S. pimpinellifolium & 275 & $1|2|$ & 28.4 & 4.I & 851 & 98 & 152 & 10 & 1.6 & 6266 \\
\hline
\end{tabular}

IAdjusted for \% polymorphic loci and average contig length of $985 \mathrm{bp}$

2Thirteeen loci have indels and no SNPs

3 Includes fresh market, processing and heirloom 
The results of our intron sequencing suggest that significant sequence diversity remains to be discovered in domesticated germplasm of tomato. These results appear to be corroborated by several studies in progress. Sequencing $48 \mathrm{COS}$ for a set of 31 landrace $\mathrm{Sl}$ lines collected from three centers of diversity, found an average SNP rate of $1 /$ 149 bp across introns and exons with $60 \%$ of the SNPs having an allele frequency below 10\% [25]. A second recent report showed that by randomly designing primers across ESTs (including exons, introns and untranslated regions) and sequencing 435 amplicons across 8 inbred lines, polymorphism ranged from $3 \%$ to $24 \%$ among $\mathrm{Sl}$ inbred lines to $63 \%$ between $S l$ inbred lines and $S$. pennellii, acc. LA716 [26]. The frequency of SNPs ranged from $10 \%$ of loci in processing lines, $11 \%$ in fresh market lines and $14 \%$ in cherry tomatoes $(S l c)$. The frequency of minor alleles (allele with lowest frequency in germplasm surveyed) in tomato breeding germplasm was below $10 \%$ for $15 / 20$ SNPs. The average frequency of minor alleles (SNPs) in our study was $18 \%$ across all the germplasm tested and $27 \%$ for breeding germplasm (See Additional file 1). The allele frequencies in our study may be higher as we pre-selected loci to be polymorphic in our germplasm and because our sampling was limited. SNPs with moderate allele frequencies (above 15\%) are particularly useful as they are informative in relevant germplasm for breeding. Conversely, rare alleles can be particularly interesting if linked or are functional alleles for rare phenotypes for genetic studies and crop improvement. Although, we chose to sequence introns of ESTs from a $\mathrm{COS}$, the frequency of polymorphisms is in a similar range as other reports $[8,14,24]$. It is not clear that COS genes would have less recombination than genes not conserved among species, especially in introns. Increasing the number and diversity of domesticated genotypes sequenced may increase the discovery of polymorphisms that can be applied to $S l$ breeding populations. The genotypes we screened (Table 2) represent genetic stocks and lines used in commercial processing and fresh market hybrids across the United States, Europe and Brazil.

In our data set, any given breeding line accounted for 11.3 to $23.8 \%$ of the polymorphism with 3-4 SNPs/locus in polymorphic loci. Considering that we prescreened 967 loci and sequenced 308 polymorphic loci (32\%), we estimate that 3.8 to $7.9 \%$ of all loci differ in elite-by-elite crosses with the set of lines sampled. $S p$ was polymorphic in $91 \%$ of loci sequenced with a SNP frequency of $1 / 851$ bp (Table 4, See Additional file 1). As only loci that were polymorphic in breeding lines were sequenced, this frequency for $S p$ is likely biased. Our estimate of polymorphism between $S p$ and $S l$ from the random set of 30 loci sequenced for all lines is $73 \%$. As we only sampled two U.S. fresh market lines, our estimates for this class may have also been biased. By cross-referencing our dataset to current resources in the Asterid family, including the updated tomato unigenes and SGN COSII dataset $[1,14]$, we have expanded the available COS in Solanaceae; provided intron sequence associated with ESTs; and diversity information relevant to loci that can form a core marker set for translational genomics research in the Solanaceae. Of the 967 loci screened, 122 including 29 SNP, 22 Indel, and 6 SSR loci have been mapped [1] (Table 1, See Additional file 1); the remaining loci are currently being mapped. These results further highlight that $S l$ has evolved considerably from $S p$ and the dilemma of application of resources built around wide crosses to elite-byelite crosses. Our dataset represents the most in depth study of variation in domesticated tomato and one of its closest progenitor species to-date.

\section{Conclusion}

Leveraging of genomic tools across species boundaries to assay variation in relevant germplasm is key to the application of these resources for crop improvement and comparative genomics. In species with limited variation due to bottlenecks from domestication and subsequent breeding, identifying sufficient polymorphism for genetic studies will require that sequence data cover more varieties and both coding and non-coding sequences. We have provided sequence data for 302 loci in 12 accessions relevant to breeding and genetic mapping in tomato These resources should enable SNP genotyping assays for highthroughput screening of germplasm for taxonomic, genetic mapping, functional analysis and breeding.

\section{Methods \\ Plant materials}

The tomato lines and species screened for this study were chosen based on their relevance to both tomato genetic research and to breeding programs. Both fresh market and processing germplasm are represented, with lines chosen to represent a diversity of environmental adaptations, market classes, parents of mapping populations, and genomic sequencing efforts. Inbred tomato lines were selected from the University of Florida and the University of North Carolina fresh market breeding programs, with selected lines of commercial interest (Table 2). Six processing lines were chosen to represent adaptation to arid (Sun1642 and M82) and humid environments (OH8245, OH88119, OH9242, and H1706); to genetic mapping (e.g. M82): and to the tomato genome sequencing project (H1706). The processing germplasm was also chosen, based on studies with molecular markers, to span sub-populations within the humid adapted germplasm (D. Francis, unpublished). Several of the processing lines are currently used as parents of hybrid varieties grown in the U.S., Brazil, and Europe. Accessions of the heirloom variety San Marzano, S. lycopersicum var. cerasiformae and a progenitor species, $S$. pimpinellifolium (Table 2) were 
selected to represent ancient varieties and closely related progenitor species. Thus the germplasm and specific polymorphisms are expected to be relevant to multiple genetic and plant improvement applications. All accessions represent inbred lines, thus should be homozygous for the majority of the loci.

\section{Defining intron position}

Intron positions were predicted by comparing COS ESTs to the Arabidopsis protein database[18] which also defines the coordinates for intron positions in Arabidopsis. We used GenBank Parser [27] to extract of intron/exon information from Arabidopsis GenBank files and compile a protein dataset with known intron/exon positions. Individual COS ESTs were then queried using BLASTX against the protein database. Predicted intron positions in tomato ESTs, the best Arabidopsis hit and intron size in Arabidopsis are displayed graphically, using an Extended Mode add-on to the Contig Viewer Program [28].

\section{Primer design and screening}

A database was established representing a COS of 1,704 tomato unigenes $(1612 \mathrm{Sl}, 29 \mathrm{~S}$. habrochaites and $63 \mathrm{~S}$. pennellii) from 113,932 ESTs [15]. From these, a single and longest EST was chosen to design primers. Using the tools developed for Compositae Genome database, the position of introns was first estimated using the procedures above. A set of 1,268 primers were designed to amplify across estimated intron sites with primers 50-100 bp from the intron. Amplification of primers was tested on a single line, M82.

Primers that successfully amplified a product were tested for polymorphism using sequencing in a series of three pools representing different degrees of diversity. The design has complementary pools representing each class (fresh market, processing, other) with one diverse line from an alternate class to maximize the chance of detecting a polymorphism within or among pools. Using a series of empirical tests with lines with known SNPs in ratios of $1: 7,1: 5,1: 3$ and $1: 1$, we determined that an unknown polymorphism can be reliably detected with sequencing with a 1:3 dilution. Pool 1 consisted of $\mathrm{O}$ 9242, FL7600, Ha7998, PI114490; Pool 2 included M82, O 8245, O 88119, NC84173 and; Pool 3 consisted of Sun1642, Heinz1706, O 9242, FL7600 (Table 2). DNA was extracted from each line and was combined in equimolar concentrations.

For all sequencing reactions, forward and reverse primers were tailed with M13 sequences and sequenced using standard protocols for Sanger sequencing (Applied Biosystems, Foster City, CA) in forward and reverse directions using a ABI 3730 (Applied Biosystems, Foster City, CA). Trace files were trimmed with Phred options -trim_cutoff
0.02" which translates to Phred 17 score. [29]. Assembly was achieved with Phrap/Consed and options were set at " -retainduplicates and -forcelevel 5". These options were optimized to give the best trim and assembly parameters for calling SNPs. Stringent trim parameters are favored in this case to minimize the high number of false SNPs associated with poor sequence on the ends. Amplicon sizes were estimated and included in Additional file 1, Tables S2 and S3. To calculate a more accurate estimate than from gel electrophoresis, the sequenced contig(s) size was used as a minimum. When greater than one contig per locus was obtained as a result of unpredictably large introns, the forward and reverse contig sizes were added.

SNPs were first identified semi-manually using Polyphred as heterozygotes within pools or homozygous differences among pools. The line, M82, was used as reference to screen amplicons for single copy number. Amplicons with putative SNPs were then amplified in the individual 12 lines (Table 2) and sequenced as described above. Only SNPs showing both homozygous alleles were called. Data was extracted from Polyphred using custom scripts ([30] See Additional file 1). Similarly, data for indels were extracted from Polyphred. SSRs (di to tetra repeats) were extracted from all sequenced loci for M82, our reference line, and the various genotypes and reported for all sequenced individuals. The sequence database was analyzed for all known repeats for tomato [1]. All loci were cross-referenced to the SGN COSII for tomato, pepper, potato and coffee and associated maps [14].

\section{List of abbreviations}

BAC: bacterial artificial chromosome

COS: conserved orthologous set

EST: expressed sequence tag

Indel: insertion/deletion

Sl: Solanum lycopersicum

Slc: Solanum lycopersicum var cerasiformae

SNP: single nucleotide polymorphism

Sp: Solanum pimpinellifolium

SSR: simple sequence repeat

\section{Authors' contributions}

Allen Van Deynze. As PI, designed, supervised and analyzed the research with co-PIs. 
Kevin Stoffel. Carried out and refined the approaches and preliminary analysis for the research.

\section{Robin Buell. Carried out the analysis of SSRs}

Jia Liu. Carried out the analysis of SSRs

Alexander Kozik. Bioinformatics analysis of source data and new sequence data

Esther van der Knaap. Designed research, discussed approaches and data and selection of germplasm

David Francis. Designed research, discussed approaches and data and selection of germplasm

All authors have read and approved the final manuscript

\section{Additional material}

\section{Additional file 1}

Table S1. Cross-reference of 1704 UC Davis COS to Tomato0607 unigene build and SGN COS II[14]. Table S2. SNP genotypes for 12 lines in tomato and 302 UCDCOS ESTs. Table S3. Indel genotypes for 12 lines in tomato and 153 UCD COS ESTs. Table S4. Polymorphisms due to SNPs (a) and Indels (b) among lines. Table S5. SSR genotypes for 12 lines in tomato and 44 UCD COS ESTs. All raw sequences have been submitted to GenBank (\#s ET165605 to ET166001).

Click here for file

[http://www.biomedcentral.com/content/supplementary/1471-

2164-8-465-S1.xls]

\section{Acknowledgements}

The authors would like to thank Mr. Brandon Tearse for converting raw polymorphism data to spreadsheet[26] format. The authors would like to thank USDA NRI/CSREES for funding this research under grant 200401575

\section{References}

I. Mueller LA, Tanksley S: Solanaceae Genomics Network. [http:/ /www.sgn.cornell.edu/].

2. Tanksley SD, Ganal MW, Prince JP, de Vicente MC, Bonierbale MW, Broun P, Fulton TM, Giovanonni JJ, Grandillo S, Martin GB, Messeguer R, Miller JC, Miller L, Paterson AH, Pineda O, Roder M, Wing RA, Wu $W$, Young ND: High density molecular linkage maps of the tomato and potato genomes. Genetics 1992, 132:1 I41-II60.

3. Kabelka E, Yang WC, Francis DM: Improved tomato fruit color within an inbred backcross line derived from Lycopersicon esculentum and L-hirsutum involves the interaction of loci. J Am Soc Hortic Sci J Am Soc Hortic Sci 2004, I 29(2):250-257.

4. Frary A, Nesbitt TC, Frary A, Grandillo S, van der Knaap E, Cong B, Liu JP, Meller J, Elber R, Alpert KB, Tanksley SD: fw2.2: A quantitative trait locus key to the evolution of tomato fruit size. Science 2000, 289(5476):85-88.

5. Fridman E, Pleban T, Zamir D: A recombination hotspot delimits a wild-species quantitative trait locus for tomato sugar content to $\mathbf{4 8 4}$ bp within an invertase gene. Proc Nat Acad Sci (USA) 2000, 97:47| 8-4723.

6. Grandillo S, Zamir D, Tanksley S: Genetic improvement of processing tomatoes: A 20 years perspective. Euphytica 1999, I I 0(2):85-97.
7. Labate JA, Baldo AM: Tomato SNP discovery by EST mining and resequencing. Molecular Breeding 2005, 16(4):343-349.

8. Yang W, Bai X, Kabelka E, Eaton C, Kamoun S, van der Knaap E, D. $F$ : Discovery of single nucleotide polymorphisms in Lycopersicon esculentum by computer aided analysis of expressed sequence tags. Molecular Breeding 2004, 14:2I-24.

9. Ching A, Caldwell KS, Jung M, Dolan M, Smith OS, Tingey S, Morgante $M$, Rafalski AJ: SNP frequency, haplotype structure and linkage disequilibrium in elite maize inbred lines. BMC Genet 2002, 3(1): 19 .

10. Van Deynze AE, Wilkins TA, Stoffel K, Lee M, Stelly D, Kozik A: A Set Of Informative Markers Designed Specifically For Breeding Cotton. In Plant and Animal Genome XV San Diego, CA. , Scherago International; 2007.

II. Fourmann M, Barret P, Froger N, Baron C, Charlot F, Delourme R, Brunel D: From Arabidopsis thaliana to Brassica napus: development of amplified consensus genetic markers (ACGM) for construction of a gene map. Theor Appl Genet 2002, 105(8): I I 96-1 206.

12. Fulton TM, Van der Hoeven R, Eannetta NT, Tanksley SD: Identification, analysis, and utilization of conserved ortholog set markers for comparative genomics in higher plants. The Plant cell 2002, I 4(7): | $457-1467$.

13. Lyons LA, Laughlin TF, Copeland NG, Jenkins NA, Womack JE, OBrien SJ: Comparative anchor tagged sequences (CATS) for integrative mapping of mammalian genomes. Nature Genetics 1997, I 5(I):47-56.

14. Wu FN, Mueller LA, Crouzillat D, Petiard V, Tanksley SD: Combining bioinformatics and phylogenetics to identify large sets of single-copy orthologous genes (COSII) for comparative, evolutionary and systematic studies: $A$ test case in the euasterid plant clade. Genetics 2006, 174(3): I407-1420.

15. Kozik A, Michelmore R: Compositae Genome Project Database - [http://cgpdb.ucdavis.edu/COS Arabidopsis/].

16. Volker B, Lushbough C, Lawrence C: PlantGDB: Resources for Plant Comparative Genomics. [http://www.plantgdb.org/pri/ ESTCluster/progress.php].

17. Timms L, Jimenez R, Chase M, Lavelle D, McHale L, Kozik A, Lai Z, Heesacker A, Knapp S, Rieseberg L, Michelmore R, Kesseli R: Analyses of synteny between Arabidopsis thaliana and species in the Asteraceae reveal a complex network of small syntenic segments and major chromosomal rearrangements. Genetics 2006, I73(4):2227-2235.

18. National Center for Biological Information. Arabidopsis thaliana FTP [ftp://ftp.ncbi.nih.gov/genomes/Arabidopsis thaliana]

19. Hong X, Scofield DG, Lynch M: Intron Size, Abundance, and Distribution within Untranslated Regions of Genes. Mol Biol Evol 2006, 23( I 2):2392-2404.

20. Pichersky E, Tanksley S: Chloroplast DNA-sequences integrated into an intron of a tomato nuclear gene. Molecular \& General Genetics 1988, 215(1):65-68.

21. Mueller LA: unirepeats.30.20060602. [ftp://ftp.sgn.cornell.edu/ tomato genome/repeats/].

22. Hsia AP, Wen TJ, Chen HD, Liu Z, Yandeau-Nelson MD, Wei Y, Guo L, Schnable PS: Temperature gradient capillary electrophoresis (TGCE)--a tool for the high-throughput discovery and mapping of SNPs and IDPs. Theor Appl Genet 2005, III(2):2। 8-225.

23. National Center for Biological Information: GenBank [http:/ /www.ncbi.nlm.nih.gov]

24. Nesbitt TC, Tanksley SD: Comparative sequencing in the genus Lycopersicon: Implications for the evolution of fruit size in the domestication of cultivated tomatoes. Genetics Ist edition. 2002, 162:365-379.

25. Labate JA, Robertson L, Sheffer SM, Wu F, Tanksley S, Baldo AM: DNA Polymorphism Estimates Within Domesticated Tomato. In PAA-Solanaceae 2006: Genomics Meets Diversity Madison, WI ; 2006.

26. Ganal MW, Luerssen H, Polley A, Wolfe M: High-throughput SNP identification and validation in tomato and pepper. In Solanaceae Genomics Conference Edited by: Spooner D. Madison, WI ; 2006:pI59.

27. Kozik A, Michelmore R: GenBank Parser. [http://www.atgc.org/ GenBankParser].

28. Kozik A, Michelmore R: Compositae Genome Python Viewer. [http://cgpdb.ucdavis.edu/SNP Discovery/Py ContigViewer/]. 
29. Ewing B, Green P: Base-calling of automated sequencer traces using phred. II. Error probabilities. Genome research 1998, 8(3): $186-194$.

30. Tearse B: Polyphred to Excel converter. [http://den drome.ucdavis.edu/wiki/doku.php?id=public:software:base].

31. Eshed Y, Zamir D: An introgression line population of Lycopersicon pennellii in the cultivated tomato enables the identification and fine mapping of yield-associated QTL. Genetics 1995, |41: I|47-I|62.

32. Berry SZ, Gould WA, Wiese KL: "Ohio 8245' Processing tomato. Hortscience 1991, 26:1093.

33. Scott JW, Jones JP: Monogenic resistance in tomato to Fusarium oxysporum f. sp. lycopersici race 3. 1989, 40:49-53.

34. van der Knaap E, Tanksley SD: Identification and characterization of a novel locus controlling early fruit development in tomato. Theoretical and Applied Genetics 200I, I03(2-3):353-358.

35. Mueller LA, Solow TH, Taylor N, Skwarecki B, Buels R, Binns J, Lin C, Wright MH, Ahrens R, Wang Y, Herbst EV, Keyder ER, Menda N, Zamir D, Tanksley SD: The SOL Genomics Network: a comparative resource for Solanaceae biology and beyond. Plant physiology 2005, I38(3): 1310-1317.

36. Omnikowski R: Pedigree of variety Heinz 1706. Report of the Tomato Genetics Cooperative 2004, 54:27.

37. Gardner RG: 'Mountain Spring' tomato; NC 8276 and NC 84173 tomato breeding lines. HortScience 1992, 27:1233-1234.

Publish with Biomed Central and every scientist can read your work free of charge

"BioMed Central will be the most significant development for disseminating the results of biomedical research in our lifetime. "

Sir Paul Nurse, Cancer Research UK

Your research papers will be:

- available free of charge to the entire biomedical community

- peer reviewed and published immediately upon acceptance

- cited in PubMed and archived on PubMed Central

- yours - you keep the copyright

Submit your manuscript here:

http://www.biomedcentral.com/info/publishing_adv.asp
BiolMedcentral 